\title{
Finite extensions of mappings from a smooth variety
}

\author{
by MAREK KARAŚ (Kraków)
}

\begin{abstract}
Let $V, W$ be algebraic subsets of $\mathbf{k}^{n}, \mathbf{k}^{m}$ respectively, with $n \leq m$. It is known that any finite polynomial mapping $f: V \rightarrow W$ can be extended to a finite polynomial mapping $F: \mathbf{k}^{n} \rightarrow \mathbf{k}^{m}$. The main goal of this paper is to estimate from above the geometric degree of a finite extension $F: \mathbf{k}^{n} \rightarrow \mathbf{k}^{n}$ of a dominating mapping $f: V \rightarrow W$, where $V$ and $W$ are smooth algebraic sets.
\end{abstract}

1. Introduction. Let $\mathbf{k}$ be any algebraically closed field of characteristic zero and $V, W$ be algebraic subsets of $\mathbf{k}^{n}, \mathbf{k}^{m}$, respectively, with $n \leq m$. It is known (under the assumption that $\mathbf{k}$ is an infinite field) that any finite mapping $f: V \rightarrow W$ can be extended to a finite mapping $F: \mathbf{k}^{n} \rightarrow \mathbf{k}^{m}$ (see $[6])$. There is the following natural problem: to estimate from above the number gdeg $F$ (geometric degree of $F$ ) of points in the "generic fiber" of the best extension $F$ (with gdeg $F$ minimal). The author ([3]-[5]) solved this problem in a few cases.

If $n=m$ and $\operatorname{gdeg} F=1$, then $F: \mathbf{k}^{n} \rightarrow \mathbf{k}^{n}$ is an isomorphism. Thus the answer to the above question is very important.

In this paper we consider a finite mapping from a smooth algebraic set such that the image is also a smooth algebraic set. We will show

TheOREM 4.4. Let $V, W \subset \mathbb{C}^{n}$ be smooth algebraic sets and let $f: V$ $\rightarrow W$ be a finite dominating mapping that is dominating on every irreducible component. If $\operatorname{dim} V=\operatorname{dim} W=k$ and $4 k+2 \leq n$, then there exists a finite mapping $F: \mathbb{C}^{n} \rightarrow \mathbb{C}^{n}$ such that $\left.F\right|_{V}=f$ and

$$
\operatorname{gdeg} F \leq(\operatorname{gdeg} f)^{2 k+1} \text {. }
$$

This is a generalization of the main Theorem of [2] which was the answer to the problem of extension of embeddings into affine space. The problem was set by S. S. Abhyankar [1].

2000 Mathematics Subject Classification: Primary 14R99; Secondary 14E25. Key words and phrases: finite mapping, geometric degree, finite extension. Research partially supported by FNP (Polish Science Foundation). 
2. Preliminaries. The coordinate ring of the algebraic set $V$ will be denoted by $\mathbf{k}[V]$. For any polynomial mapping $f=\left(f_{1}, \ldots, f_{n}\right): V \rightarrow W$ the homomorphism of the coordinate rings $\mathbf{k}[W] \ni P \mapsto P\left(f_{1}, \ldots, f_{n}\right) \in \mathbf{k}[V]$ will be denoted by $f^{*}$.

Definition 2.1. A polynomial mapping $f: V \rightarrow W$ is called finite if $\mathbf{k}[V]$ is an integral extension of the ring $f^{*}(\mathbf{k}[W])=\mathbf{k}\left[f_{1}, \ldots, f_{m}\right]$.

Finite mappings have the following properties:

TheOREm 2.2. (1) If $f$ is finite, then there exists a number $d \in \mathbb{N}$ such that $\# f^{-1}(y) \leq d$ for all $y \in W$.

(2) If $\mathbf{k}=\mathbb{C}$, then $f$ is finite iff $f$ is proper (in the natural topology).

(3) If $f, g$ are finite, then $g \circ f$ is finite.

(4) If $f, g$ are polynomial mappings and $g \circ f$ is finite, then $f$ is finite. If , moreover, $f$ is dominating, then $g$ is finite.

(5) If $f$ is finite and dominating, then $f$ is surjective.

(6) If $f: V \rightarrow W$ is finite and $Z$ is an algebraic subset of $V$, then $\left.f\right|_{Z}$ is finite.

(7) If $f: V \rightarrow W$ is finite and $Z$ is an algebraic subset of $V$, then $\operatorname{dim} f(Z)=\operatorname{dim} Z$.

(8) If $f_{i}: V_{i} \rightarrow W_{i}$ are finite for $i=1,2$, and $\left.f_{1}\right|_{V_{1} \cap V_{2}}=\left.f_{2}\right|_{V_{1} \cap V_{2}}$, then $f_{1} \cup f_{2}$ is finite.

P r o of. Assertions (1), (3), (4), (6) and (8) are easy consequences of the definition. For the proof of (2) see e.g. [8]. The proof of (5) can be found e.g. in [9] (Thm. I.5.5), and (7) is a consequence of the definition of dimension.

If $f: V \rightarrow W$ is dominating then $f^{*}: \mathbf{k}[W] \rightarrow \mathbf{k}[V]$ is a monomorphism. In this case we will identify $\mathbf{k}[W]$ with $f^{*}(\mathbf{k}[W]) \subset \mathbf{k}[V]$. If $V$ and $W$ are irreducible, then $\mathbf{k}[W]$ and $\mathbf{k}[V]$ are integral domains and therefore $f^{*}$ can be extended to a monomorphism $f^{*}: \mathbf{k}(W) \rightarrow \mathbf{k}(V)$ of fields. In the same way as before we will identify $\mathbf{k}(W)$ with $f^{*}(\mathbf{k}(W)) \subset \mathbf{k}(V)$.

We have the following

Theorem 2.3 (see e.g. [7], Thm. 3.17). Let $V \subset \mathbf{k}^{n}$ and $W \subset \mathbf{k}^{m}$ be irreducible algebraic sets of the same dimension. If $f: V \rightarrow W$ is dominating then there exists an open and dense subset $U \subset W$ such that

$$
\# f^{-1}(y)=[\mathbf{k}(V): \mathbf{k}(W)] \quad \text { for } y \in U .
$$

In [7] Theorem 2.3 is stated for $\mathbf{k}=\mathbb{C}$ but the proof given there works for an algebraically closed field of characteristic zero.

From Theorem 2.3 and the theorem about the dimension of the fibers (see e.g. [9], Thm. I.6.7) we have 
LEMMA 2.4. Let $V \subset \mathbf{k}^{n}$ and $W \subset \mathbf{k}^{m}$ be algebraic sets. If $W$ is irreducible and $f: V \rightarrow W$ dominating then there is an open and dense set $U \subset W$ such that the function $U \ni y \mapsto \# f^{-1}(y) \in \mathbb{N} \cup\{\infty\}$ is constant.

Proof. Let $V=V_{1} \cup \ldots \cup V_{r}$ be the decomposition of $V$ into irreducible components. We have $\overline{f(V)}=\overline{f\left(V_{1}\right)} \cup \ldots \cup \overline{f\left(V_{r}\right)}$ and, since $W$ is irreducible, $\overline{f\left(V_{i}\right)}=W$ for some $i \in\{1, \ldots, r\}$.

Let $W^{*}$ be the union of those $\overline{f\left(V_{i}\right)}$ for which $\overline{f\left(V_{i}\right)} \neq W$. Since $W$ is irreducible, $W \backslash W^{*}$ is an open and dense subset of $W$ for which $f^{-1}\left(W \backslash W^{*}\right) \subset$ $\bigcup_{i \in I} V_{i}$, where $I:=\left\{i \in\{1, \ldots, r\} \mid \overline{f\left(V_{i}\right)}=W\right\}$. We may assume that $I=\{1, \ldots, k\}$ for some $k \leq r$. By Theorem 2.3 for all $i=1, \ldots, k$ there exist $U_{i} \subset W$ open and dense in $W$ such that

$$
\#\left(\left.f\right|_{V_{i}}\right)^{-1}(y)=\left[\mathbf{k}\left(V_{i}\right): \mathbf{k}(W)\right] \quad \text { for } y \in U_{i} .
$$

For the set $\widetilde{U}:=U_{1} \cap \ldots \cap U_{k}$, which is also open and dense, we infer that

$$
\#\left(\left.f\right|_{V_{i}}\right)^{-1}=\left[\mathbf{k}\left(V_{i}\right): \mathbf{k}(W)\right] \quad \text { for } y \in \widetilde{U}, i=1, \ldots, k .
$$

For $i \in\{1, \ldots, k\}$ set $V_{i}^{*}:=V_{i} \cap \bigcup_{j \neq i} V_{j}$. Since $V_{i}^{*} \neq V_{i}$ and $V_{i}$ is irreducible, we see that $\operatorname{dim} V_{i}^{*}<\operatorname{dim} V_{i}$ and $\overline{f\left(V_{i}^{*}\right)} \neq W$.

By irreducibility of $W$ we get $\overline{f\left(V_{1}^{*}\right)} \cup \ldots \cup \overline{f\left(V_{k}^{*}\right)} \neq W$, and consequently $U^{*}:=W \backslash\left(\overline{f\left(V_{1}^{*}\right)} \cup \ldots \cup \overline{f\left(V_{k}^{*}\right)}\right)$ is open and dense in $W$.

Now we have

$$
\#\left(\left.f\right|_{V_{1} \cup \ldots \cup V_{k}}\right)^{-1}(y)=\sum_{i=1}^{k}\left[\mathbf{k}\left(V_{i}\right): \mathbf{k}(V)\right] \quad \text { for } y \in \widetilde{U} \cap U^{*},
$$

where $\widetilde{U} \cap U^{*}$ is open and dense in $W$. Finally $U:=\widetilde{U} \cap U^{*} \cap\left(W \backslash W^{*}\right)$ is an open and dense subset of $W$ such that

$$
\# f^{-1}(y)=\sum_{i=1}^{k}\left[\mathbf{k}\left(V_{i}\right): \mathbf{k}(V)\right] \quad \text { for } y \in U
$$

Now we can state

Definition 2.5. Let $f: V \rightarrow W$ be a dominating polynomial mapping. If $W$ is an irreducible set then the constant number of points in the fibers of $f$ on the set $U$ (see Lemma 2.4) is called the geometric degree of the mapping $f$ and denoted by gdeg $f$. If the set $W$ is reducible then

$$
\begin{aligned}
& \operatorname{gdeg} f:=\max \left\{\operatorname{gdeg}\left(\left.f\right|_{f^{-1}(Z)}\right) \mid Z \subset W\right. \\
&\text { is an irreducible component of } W\} .
\end{aligned}
$$

For any polynomial mapping we can define its geometric degree by putting $W=\overline{f(V)}$. In particular we can define the geometric degree for any finite mapping. 
In the general case there may exists $y \in W$ with $\# f^{-1}(y)>\operatorname{gdeg} f$. This is illustrated by

ExAmple 2.6. Let $F: \mathbb{C} \ni t \mapsto\left(t^{2}-1, t\left(t^{2}-1\right)\right) \in \mathbb{C}^{2}$. Then $F(\mathbb{C})=W$, where $W=\left\{(x, y) \in \mathbb{C}^{2} \mid y^{2}=x^{2}+x^{3}\right\}$ and gdeg $F=1$, because $F$ is a parametrization of the rational curve $W$, but $F^{-1}((0,0))=\{-1,1\}$. If we take $f=\left.F\right|_{\{-1,1\}}$, then we obtain a finite mapping $f:\{-1,1\} \rightarrow \mathbb{C}^{2}$ with extension $F$ and gdeg $f=2>\operatorname{gdeg} F$.

The following theorem gives a condition under which it is impossible to find a point $y \in W$ with $\# f^{-1}(y)>\operatorname{gdeg} f$.

Theorem 2.7 (see e.g. [9], Thm. II 5.6). If $f: V \rightarrow W$ is finite and dominating, $V$ and $W$ are irreducible and $W$ is normal then

$$
\# f^{-1}(y) \leq \operatorname{gdeg} f \quad \text { for } y \in W \text {. }
$$

3. Extensions of projections. In [4] we proved

Theorem 3.1 ([4], Thm. 3.10). Let $V \subset \mathbf{k}^{k} \times \mathbf{k}^{n}$ be an irreducible algebraic set, and $\pi: V \rightarrow 0 \times \mathbf{k}^{n}$ the natural projection. If $\pi$ is finite and $\pi(V)$ is normal then there exists a finite mapping $\Pi: \mathbf{k}^{k} \times \mathbf{k}^{n} \rightarrow \mathbf{k}^{k} \times \mathbf{k}^{n}$ such that $\left.\Pi\right|_{V}=\pi$ and

$$
\operatorname{gdeg} \Pi \leq(\operatorname{gdeg} \pi)^{k} .
$$

Here, we prove a slight generalization (Theorem 3.6). A reducible set is meant to be normal if each of its points is normal.

First we define an auxiliary notion.

Definition 3.2. A polynomial mapping $f: V \rightarrow W$ is called dominating on the irreducible component $V^{\prime} \subset V$ if $\overline{f\left(V^{\prime}\right)}$ is an irreducible component of $W$.

ExAmple 3.3. Let $V=\left\{(x, y) \in \mathbb{C}^{2} \mid x y=0\right\}, W=\mathbb{C}$ and $f: V \ni$ $(x, y) \mapsto x \in W$. The mapping $f$ is dominating and dominating on the component $\left\{(x, y) \in \mathbb{C}^{2} \mid y=0\right\}$ but it is not dominating on the component $\left\{(x, y) \in \mathbb{C}^{2} \mid x=0\right\}$.

EXAmple 3.4. Let $V=W=\left\{(x, y) \in \mathbb{C}^{2} \mid x y=0\right\}$ and $f: v \ni(x, y) \mapsto$ $(x+y, 0) \in W$. The mapping $f$ is dominating on every component of $V$ but it is not dominating.

REMARK 3.5. If $f: V \rightarrow W$ is finite and dominating, then it is dominating on every component of maximal dimension.

THEOREM 3.6. Let $V \subset \mathbf{k}^{k} \times \mathbf{k}^{n}$ be an algebraic set, and let $\pi: V \rightarrow$ $0 \times \mathbf{k}^{n}$ be the natural projection. If $\pi: V \rightarrow \pi(V)$ is finite and dominating 
on every component, and $\pi(V)$ is normal, then there exists a finite mapping $\Pi: \mathbf{k}^{k} \times \mathbf{k}^{n} \rightarrow \mathbf{k}^{k} \times \mathbf{k}^{n}$ such that $\left.\Pi\right|_{V}=\pi$ and

$$
\operatorname{gdeg} \Pi \leq(\operatorname{gdeg} \pi)^{k} \text {. }
$$

Proof. Let $V=V_{1} \cup \ldots \cup V_{r}$ and $W=W_{1} \cup \ldots \cup W_{s}$ be decompositions into irreducible components. Because $W$ is normal, $W_{i} \cap W_{j}=\emptyset$ for $i \neq j$. We have

$$
\pi^{-1}\left(W_{1}\right)=\bigcup_{i \in I_{1}} V_{i}, \quad \ldots, \quad \pi^{-1}\left(W_{s}\right)=\bigcup_{i \in I_{s}} V_{i}
$$

where $I_{1} \cup \ldots \cup I_{s}=\{1, \ldots, r\}$ and $I_{i} \cap I_{j}=\emptyset$ for $i \neq j$. For any $l \in\{1, \ldots, s\}$ consider the mapping

$$
\pi_{l}=\left.\pi\right|_{\pi^{-1}\left(W_{l}\right)}: \pi^{-1}\left(W_{l}\right) \rightarrow W_{l} .
$$

By Definition 2.5 we have

$$
\operatorname{gdeg} \pi_{l} \leq \operatorname{gdeg} \pi
$$

The mapping $f$ is dominating on every component, so

$$
\operatorname{gdeg} \pi_{l}=\sum_{j \in I_{l}}\left[\mathbf{k}\left(V_{j}\right): \mathbf{k}\left(W_{l}\right)\right]
$$

(see the proof of Lemma 2.4). For any $j \in I_{l}$ and $i \in\{1, \ldots, k\}$ let $H_{i, j, l} \in$ $\mathbf{k}\left(W_{l}\right)[T]$ be the minimal monic polynomial for $\left.x_{i}\right|_{V_{j}}$ (restriction of $x_{i}$ to the set $V_{j}$ ) over the field $\mathbf{k}\left(W_{l}\right)$, where $x_{1}, \ldots, x_{k}$ and $y_{1}, \ldots, y_{n}$ are coordinates in $\mathbf{k}^{k}$ and $\mathbf{k}^{n}$ respectively. We have $\operatorname{deg} H_{i, j, l} \leq\left[\mathbf{k}\left(V_{j}\right): \mathbf{k}\left(W_{l}\right)\right]$. The element $\left.x_{i}\right|_{V_{J}}$ is integral over the ring $\mathbf{k}\left[W_{l}\right]$. The ring $\mathbf{k}\left[W_{l}\right]$ is normal, so $H_{i, j, l} \in$ $\mathbf{k}\left[W_{l}\right][T]$. Now we put

$$
H_{i, l}=\prod_{j \in I_{l}} H_{i, j, l} .
$$

The polynomial $H_{i, l}$ is monic and

$$
H_{i, l}\left(x_{i}\right)=0 \quad \text { on } \bigcup_{j \in I_{l}} V_{j}
$$

Also

$$
\operatorname{deg} H_{i, l}=\sum_{j \in I_{l}} \operatorname{deg} H_{i, j, l} \leq \sum_{j \in I_{l}}\left[\mathbf{k}\left(V_{j}\right): \mathbf{k}\left(W_{l}\right)\right]=\operatorname{gdeg} \pi_{l} \leq \operatorname{gdeg} \pi .
$$

Multiplying $H_{i, l}$, if necessary, by some power of $T$, we can assume that $\operatorname{deg} H_{i, l}=\operatorname{gdeg} \pi$. Thus we can write

$$
H_{i, l}=T^{d}+a_{i, l, d-1} T^{d-1}+\ldots+a_{i, l, 0}
$$

where $d=\operatorname{gdeg} \pi$ and $a_{i, l, j} \in \mathbf{k}\left[W_{l}\right]$. The functions $a_{i, j}=a_{i, 1, j} \cup \ldots \cup a_{i, s, j}$ are regular on $W_{1} \cup \ldots \cup W_{s}=W$. Thus

$$
H_{i}=T^{d}+a_{i, d-1} T^{d-1}+\ldots+a_{i, 0}
$$

is a monic polynomial in $\mathbf{k}[W][T]$ such that

$$
H_{i}\left(x_{i}\right)=0 \quad \text { on } V \text {. }
$$


Let $G_{i}$ be a polynomial of $\mathbf{k}\left[y_{1}, \ldots, y_{n}\right][T]$ obtained from $H_{i}$ by replacing $\left.y_{i}\right|_{W}$ with $y_{i}$ and

$$
\Pi: \mathbf{k}^{k} \times \mathbf{k}^{n} \ni(x, y) \mapsto\left(G_{1}\left(x_{1}, y\right), \ldots, G_{k}\left(x_{k}, y\right), y\right) \in \mathbf{k}^{k} \times \mathbf{k}^{n} .
$$

Because $H_{i}\left(\left.x_{i}\right|_{V}\right)=0$, we have $\left.\Pi\right|_{V}=\pi$. Furthermore $\Pi$ is finite. Indeed, $\mathbf{k}\left[x_{1}, \ldots, x_{k}, y_{1}, \ldots, y_{n}\right]$ is an integral extension of $\mathbf{k}\left[G_{1}\left(x_{1}, y\right), \ldots\right.$ $\left.\ldots, G_{k}\left(x_{k}, y\right), y_{1}, \ldots, y_{n}\right]$ because

$$
G_{i}(T, y)-G_{i}\left(x_{i}, y\right) \in \mathbf{k}\left[G_{1}\left(x_{1}, y\right), \ldots, G_{k}\left(x_{k}, y\right), y_{1}, \ldots, y_{n}\right][T]
$$

is a monic polynomial which vanishes at $x_{i}$.

Finally, for each $\left(x^{\prime}, y^{\prime}\right) \in \mathbf{k}^{k} \times \mathbf{k}^{n}$ we have

$$
\begin{aligned}
& \#\left\{(x, y) \in \mathbf{k}^{k} \times \mathbf{k}^{n}: \Pi(x, y)=\left(x^{\prime}, y^{\prime}\right)\right\} \\
& =\#\left\{(x, y) \in \mathbf{k}^{k} \times \mathbf{k}^{n}: y=y^{\prime}, G_{i}\left(x_{i}, y\right)=x_{i}^{\prime} \text { for } i=1, \ldots, k\right\} \\
& \leq \operatorname{deg} G_{1} \cdot \ldots \cdot \operatorname{deg} G_{k} \leq(\operatorname{gdeg} \pi)^{k} .
\end{aligned}
$$

Thus $\Pi$ is a finite extension of $\pi$ such that gdeg $\Pi \leq(\operatorname{gdeg} \pi)^{k}$.

An example showing that the normality of $\pi(V)$ is necessary in Theorems 3.1 and 3.6 can be found in [4].

4. Proof of the main result. Let us recall some facts about embeddings.

Definition 4.1. A polynomial mapping $f: V \rightarrow \mathbf{k}^{n}$ is called an embedding if $f$ is an isomorphism onto its image $f(V)=\overline{f(V)}$.

We have the following well known lemma (see e.g. [2]):

Lemma 4.2. If $X \subset \mathbb{C}^{n}$ is a closed algebraic smooth set, $\operatorname{dim} X=k$ and $n>2 k+1$, then we can change coordinates in such a way that the projection

$$
\phi: X \ni(x, y) \mapsto(0, y) \in 0 \times \mathbb{C}^{2 k+1}
$$

is an embedding.

We also have

Theorem 4.3 ([2], Thm. 1.2). Let $X \subset \mathbb{C}^{n}$ be a closed algebraic set which is smooth and not necessarily irreducible of (not necessarily pure) dimension $k$. Let $\phi: X \rightarrow \mathbb{C}^{n}$ be an embedding. If $n \geq 4 k+2$ then there exists an isomorphism $\Phi: \mathbb{C}^{n} \rightarrow \mathbb{C}^{n}$ such that $\left.\Phi\right|_{X}=\phi$.

Now we are in a position to prove the main result.

THEOREM 4.4. Let $V, W \subset \mathbb{C}^{n}$ be smooth algebraic sets, and let $f: V$ $\rightarrow W$ be a finite dominating mapping that is dominating on every irreducible component. If $\operatorname{dim} V=\operatorname{dim} W=k$ and $4 k+2 \leq n$, then there exists a finite mapping $F: \mathbb{C}^{n} \rightarrow \mathbb{C}^{n}$ such that $\left.F\right|_{V}=f$ and

$$
\operatorname{gdeg} F \leq(\operatorname{gdeg} f)^{2 k+1} \text {. }
$$


Proof. By Lemma 4.2 we can assume that the projections

$$
\phi_{1}: V \rightarrow 0 \times \mathbb{C}^{2 k+1} \text { and } \phi_{2}: W \rightarrow 0 \times \mathbb{C}^{n-2 k-1}
$$

are embeddings. Put

$$
\widetilde{V}=\phi_{1}(V), \quad \widetilde{W}=\phi_{2}(W) \text { and } \tilde{f}=\phi_{2} \circ f \circ \phi_{1}^{-1}: \widetilde{V} \rightarrow \widetilde{W} .
$$

The mapping $\tilde{f}$ is finite with $\operatorname{gdeg} \tilde{f}=\operatorname{gdeg} f$.

Because $\widetilde{V} \subset 0 \times \mathbb{C}^{2 k+1}, \widetilde{W} \subset 0 \times \mathbb{C}^{n-2 k-1}$, we can consider the sets $\widetilde{V}$ and $\widetilde{W}$ as subsets of $\mathbb{C}^{2 k+1}$ and $\mathbb{C}^{n-2 k-1}$, respectively. Consider the isomorphism

$$
\psi: \widetilde{V} \ni x \mapsto(x, \widetilde{f}(x)) \in \mathbb{C}^{2 k+1} \times \mathbb{C}^{n-2 k-1},
$$

the set $\widehat{V}=\psi(\widetilde{V})$, and the projection

$$
\pi: \widehat{V} \ni(x, y) \mapsto(0, y) \in 0 \times \mathbb{C}^{n-2 k-1} .
$$

We have $\tilde{f}=\pi \circ \psi$, and $\psi$ is an isomorphism, so $\pi$ is finite and $\operatorname{gdeg} \pi=$ $\operatorname{gdeg} \widetilde{f}=\operatorname{gdeg} f$. The set $\pi(\widehat{V})=\widetilde{W}$ is smooth, because $W$ is smooth, and by Theorem 3.6 there exists a finite mapping $\Pi: \mathbb{C}^{2 k+1} \times \mathbb{C}^{n-2 k-1} \rightarrow$ $\mathbb{C}^{2 k+1} \times \mathbb{C}^{n-2 k-1}$ such that $\left.\Pi\right|_{\widehat{V}}=\pi$ and

$$
\operatorname{gdeg} \Pi \leq(\operatorname{gdeg} \pi)^{2 k+1} \text {. }
$$

By Theorem 4.3 applied to $\psi: \widetilde{V} \rightarrow \widehat{V}, \phi_{1}: V \rightarrow \widetilde{V}$ and $\phi_{2}: W \rightarrow \widetilde{W}$ there exist isomorphisms $\Psi, \Phi_{1}, \Phi_{2}: \mathbb{C}^{n} \rightarrow \mathbb{C}^{n}$ such that

$$
\left.\Psi\right|_{\widetilde{V}}=\psi,\left.\quad \Phi_{1}\right|_{V}=\phi_{1},\left.\quad \Phi_{2}\right|_{W}=\phi_{2} .
$$

Putting $F=\Phi_{2}^{-1} \circ \Pi \circ \Psi \circ \Phi_{1}$ we have a finite extension of $f$ such that $\operatorname{gdeg} F \leq(\operatorname{gdeg} f)^{2 k+1}$.

Note that Theorem 4.4 is a generalization of Theorem 4.3. Indeed, if $f: V \rightarrow \mathbb{C}^{n}$ is an embedding and $V$ is smooth then $f(V)$ is also smooth, $f$ is a finite dominating mapping that is dominating on every component and gdeg $f=1$. By Theorem 4.4 there exists a finite mapping $F: \mathbb{C}^{n} \rightarrow \mathbb{C}^{n}$ such that $\left.F\right|_{V}=f$ and gdeg $F=1$. Now $F$ is a birational mapping, and by Zariski's Main Theorem, it is an isomorphism.

Note, also, that if $V$ is a pure dimensional set, then the assumption that $f$ is dominating on every irreducible component is not necessary (see Remark 3.5).

By Theorem 4.4 we have

COROLlaRY 4.5. Under the assumptions of Theorem 4.4 there are infinitely many finite mappings $F: \mathbb{C}^{n} \rightarrow \mathbb{C}^{n}$ such that $\left.F\right|_{V}=f$ and

$$
\operatorname{gdeg} F \leq(\operatorname{gdeg} f)^{2 k+1} \text {. }
$$

Proof. Let $x \in \mathbb{C}^{n} \backslash V$. For any point $y \in \mathbb{C}^{n} \backslash W$ we define a mapping $f_{y}: V \cup\{x\} \rightarrow W \cup\{y\}$ such that $\left.f_{y}\right|_{V}=f$ and $f_{y}(x)=y$. By Theorem 4.4 
there exists a finite mapping $F_{y}: \mathbb{C}^{n} \rightarrow \mathbb{C}^{n}$ such that $\left.F_{y}\right|_{V \cup\{x\}}=f_{y}$ and

$$
\operatorname{gdeg} F_{y} \leq\left(\operatorname{gdeg} f_{y}\right)^{2 k+1}=(\operatorname{gdeg} f)^{2 k+1} .
$$

Obviously $\left.F_{y}\right|_{V}=f$ and $F_{y} \neq F_{y^{\prime}}$ for $y \neq y^{\prime}$.

By Theorem 4.4 we also have

Corollary 4.6. Let $V, W \subset \mathbb{C}^{n}$, where $n>1$, be finite sets. For any mapping $f: V \rightarrow W$ there exists a finite mapping $F: \mathbb{C}^{n} \rightarrow \mathbb{C}^{n}$ such that $\left.F\right|_{V}=f$ and $\operatorname{gdeg} F=\operatorname{gdeg} f$.

Because Lemma 4.2 and Theorem 4.3 are true for any algebraically closed field, we have

REMARK 4.7. Theorem 4.4 holds for any algebraically closed field of characteristic zero.

\section{References}

[1] S. S. Abhyankar, On the Semigroup of a Meromorphic Curve, Kinokuniya BookStore, Tokyo, 1978.

[2] Z. Jelonek, The extension of regular and rational embeddings, Math. Ann. 277 (1987), 113-120.

[3] M. Karaś, An estimation of the geometric degree of an extension of some polynomial proper mappings, Univ. Iagell. Acta Math. 35 (1997), 131-135.

[4] -, Geometric degree of finite extensions of projections, ibid. 37 (1999), 109-119.

[5] —, Birational finite extensions, J. Pure Appl. Algebra 148 (2000), 251-253.

[6] M. Kwieciński, Extending finite mappings to affine space, ibid. 76 (1991), 151-153.

[7] D. Mumford, Algebraic Geometry I. Complex Projective Varieties, Springer, Heidelberg, 1976.

[8] K. J. Nowak, The extension of holomorphic functions of polynomial growth on algebraic sets in $\mathbb{C}^{n}$, Univ. Iagell. Acta Math. 28 (1991), 19-28.

[9] I. R. Shafarevich, Basic Algebraic Geometry, Springer, Heidelberg, 1974.

Institute of Mathematics

Jagiellonian University

Reymonta 4

30-059 Kraków, Poland

E-mail: karas@im.uj.edu.pl 